\title{
Stable single-atom contacts of zinc whiskers
}

\author{
P. Konrad, C. Bacca, and E. Scheer ${ }^{\mathrm{a})}$ \\ Fachbereich Physik, Universität Konstanz, D-78457 Konstanz, Germany \\ P. Brenner, A. Mayer-Gindner, and H. v. Löhneysen ${ }^{\text {b) }}$ \\ Physikalisches Institut, Universität Karlsruhe, D-76128 Karlsruhe, Germany
}

(Received 28 June 2004; accepted 28 March 2005; published online 20 May 2005)

\begin{abstract}
We report low-temperature measurements of the electrical transport properties of single-atom and tunnel contacts of $\mathrm{Zn}$. We present a variant of the mechanically controllable break-junction technique that comprises a whisker as the central part. This technique combines the possibility to fabricate reproducibly single-atom contacts and well-characterized electronic and lattice properties of the electrodes. From tunneling current-voltage characteristics, we deduce a well-pronounced quasiparticle spectrum for whiskers, while for standard "notched-wire" and thin-film break junctions the spectrum is smeared out. (C) 2005 American Institute of Physics. [DOI: 10.1063/1.1926408]
\end{abstract}

The tunneling density of states ${ }^{1}$ is usually deduced from electronic transport measurements through tunneling contacts, fabricated by different methods. However, although discovered many years ago, for several elementary superconductors direct transport measurements are still lacking because of the difficulty to fabricate suitable tunneling contacts. The divalent metal $\mathrm{Zn}$ is an example for this situation where, besides the metallurgical problems that hinder the creation of tunneling contacts with standard methods, the superconducting properties are anisotropic.

Single-atom or few-atom contacts are model systems to investigate the electronic transport properties on the atomic scale because the electronic conduction can be regarded as a fully quantum-mechanical scattering problem, i.e., the conductance $G$ can be attributed to independent nick-named conduction channels which contribute to the total conductance with a weight given by their transmission coefficients $\tau_{i}: G=G_{0} \sum_{i=1}^{N} \tau_{i}\left(G_{0}=2 e^{2} / h\right.$ is the conductance quantum and $N$ the number of channels). ${ }^{2}$ An atomic-size contact between two metallic electrodes can accommodate only a small number of conduction channels the number and $\tau_{i}$ of which depend on the chemical properties of the atoms forming the contact and on their geometrical arrangement. The latter information has been deduced from the fact that the transport properties change when rearranging the contact region. ${ }^{3,4}$ The use of a hexagonal metal allows to establish different configurations of the few-atom contacts in contrast to the cubic metals that have been studied so far.

In principle, all physical properties that depend on the channel ensemble $\left\{\tau_{i}\right\}$ could be used to determine the transport channels. However, independent determination of all $\tau_{i}^{\prime} \mathrm{s}$ with arbitrary values has so far only successfully been performed by analyzing current-voltage characteristics $(I-V s)$ of superconducting contacts. The analysis relies on the quantitative agreement between the calculated $I-V s$ for BCS superconductors and the experimental data. ${ }^{4,5}$ Consequently, the superconducting properties of the electrodes forming the atomic-size contact have to be of high quality.

In the present work the measurement of the $\tau_{i}^{\prime} \mathrm{s}$ is accomplished for $\mathrm{Zn}$, a metal not explored so far, by using

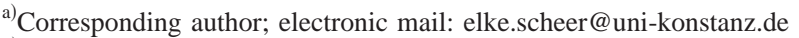

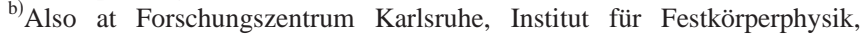
D-76021 Karlsruhe, Germany.
}

mechanically controllable break junctions (MCB) (Refs. 3 and 6) of whiskers glued on top of a prepatterned flexible substrate (see Fig. 1). Zn whiskers grow with a probability of $67 \%$ along the crystallographic $a$ axis. ${ }^{7}$

The whiskers are produced by the following method: We electroplate $\simeq 8$ to $10 \mu \mathrm{m}$ thick layers of $99.99 \%$ pure $\mathrm{Zn}$ onto $0.25 \mathrm{~mm}$ thick stainless steel substrates. In order to obtain continuous and homogeneous $\mathrm{Zn}$ layers, the surfaces of the substrates have to be prepared in three steps: (1) Sanding and subsequent cleaning of the substrates in diluted $\mathrm{HCl}$; (2) electroplating a thin $(0.8$ to $1 \mu \mathrm{m}) \mathrm{Zn}$ layer; and (3) removing the layer completely in $\mathrm{HCl}$. The final electroplating is then performed in a solution of $100 \mathrm{~g} \mathrm{ZnCl}$ per liter $\mathrm{H}_{2} \mathrm{O}$ and a current of $2 \mathrm{~A}$ for $110 \mathrm{~s}$. In order to enhance the homogeneity, the plates are turned by $180^{\circ}$ at half time. With these parameters, crystallites of $0.5-1 \mu \mathrm{m}$ in diameter and the $c$ axis almost perpendicular to the surface can be created.

A package of 15 substrates, coated on both sides, is then pressed together between stainless steel plates with stainless steel screws tightened to a torque of $20 \mathrm{Nm}$. The edges of the package are sanded and polished carefully until they have a shining surface with a roughness of less than $\sim 0.3 \mu \mathrm{m}$. The package is then stored at $T=80{ }^{\circ} \mathrm{C}$ and $p=200$ mbar helium atmosphere for several weeks. The growth seems to saturate after a period of three to six months, varying from package to package. With a higher torque of $30 \mathrm{Nm}$, a faster, but irregular growth was achieved. The diameters of the whiskers range from 0.5 to $2 \mu \mathrm{m}$ with a tendency of thicker whiskers for a lower torque. After a growth period of six

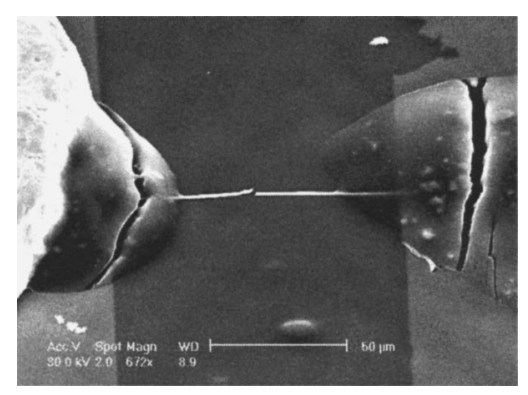

FIG. 1. Optical micrograph of a whisker MCB after measurement. The thin wire in the center is the $\mathrm{Zn}$ whisker which is glued with epoxy resin and to Au electrodes patterned on a substrate covered with an insulating layer. The two almost vertical lines with a spacing of $100 \mu \mathrm{m}$ are the edges of the $\mathrm{Au}$ electrodes. In between the insulating under-layer is visible. 
months the average length is $\sim 1.5 \mathrm{~mm}$ and a considerable amount of whiskers has grown straight without kinks. The shape of the cross section is irregular and does not correlate with the growth direction. ${ }^{7}$

For contacting and manipulating the whiskers, we prepare bronze substrates of size $3 \mathrm{~mm} \times 18 \mathrm{~mm}$ covered with an insulating layer of polyimide and $70 \mathrm{~nm}$ thick gold electrodes separated by a spacing of $100 \mu \mathrm{m}$ defined by the shadow of a thin wire. For smaller spacings no reliable breaking of the whiskers could be achieved within the elastic bending limit of the substrate. An individual whisker is chosen, picked manually by touching it slightly with a thin metal wire or a needle. It usually breaks close to the surface. Due to the adhesion it can thus be lifted from the growing block and deposited onto the substrate with the prepared electrode structure. The mechanical contact to the substrate, necessary for breaking the whisker, is provided by two small dots (diameter $\approx 50 \mu \mathrm{m}$ ) of epoxy resin close to the inner edges of the gold pads. This procedure usually reduces the freestanding length of the whisker to about 50 to $60 \mu \mathrm{m}$. The electrical contact between the whiskers and the electrodes is obtained by gluing two small pieces of In at a temperature of $120^{\circ} \mathrm{C}$ onto the whisker and the pads. Finally, the In pieces are fixed by a small amount of epoxy resin at their outer ends. With this method we obtain a total two-lead resistance of the whisker break junction at room temperature of 10 to $100 \Omega$. The low-temperature measurements (see below) demonstrate that the superconducting properties are well pronounced while for other contacting methods only a very rounded tunneling density of states could be deduced from the measurements.

A whisker MCB with a room-temperature resistance of $R(300 \mathrm{~K})=39 \Omega$ is mounted on a three-point bending mechanism ${ }^{6}$ thermally anchored to the coldest point of a ${ }^{3} \mathrm{He}$ cryostat with base temperature of $\approx 260 \mathrm{mK}$.

The residual two-lead resistance was $R=4.7 \Omega$ above the superconducting transition temperature of $T_{c} \simeq 0.8 \mathrm{~K}$ and $R_{0}=4.0 \Omega$ below it. Thus we deduce a normal-state resistance of the whisker of $R_{W}=0.7 \Omega$ (distance between the indium spots was $200 \mu \mathrm{m}$, diameter of the whisker $1 \mu \mathrm{m}$ ) that corresponds to a specific resistance of $0.3 \mu \Omega \mathrm{cm}$, somewhat higher than reported in the literature. ${ }^{8}$

The successful operation of the whisker break junction at $T=1.4 \mathrm{~K}$ is demonstrated by conductance measurements while the junction is broken or reconnected, respectively. An example of an opening curve, i.e., the conductance versus elongation of the bridge, is shown in the inset of Fig. 2. It displays the typical behavior for $\mathrm{Zn}$ atomic-size contacts that has been observed already in samples prepared by different methods: ${ }^{9,10}$ The conductance decreases stepwise but the steps and plateaus are not very well pronounced. The conductance of the last plateau, presumably corresponding to a single-atom contact is in the order of $0.8 G_{0}$. A crucial test of the stability of the device can be performed in the tunnel regime, since then the conductance varies exponentially with the distance of the electrodes. When halting the curvature of the substrate, the drift of the conductance within one hour is smaller than our measurement resolution $\simeq 10^{-3}$. We thus estimate the stability of the electrode distance to be better than $1 \mathrm{pm} / \mathrm{h}$.

The superconducting properties are probed at $T$ $=270 \mathrm{mK}$ well below $T_{c}$, where a tunnel contact with resistance $R \simeq 0.5 \mathrm{M} \Omega$ leads to a current-voltage characteristic Downloaded 12 May 2006 to 134.34.148.124. Redistribution subje

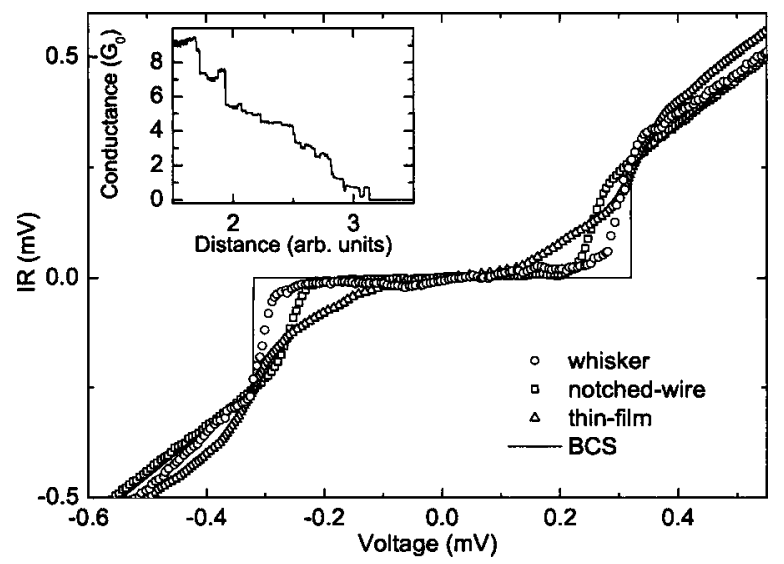

FIG. 2. Main panel: Current-voltage characteristics of tunnel contacts of $\mathrm{Zn}$ fabricated by three different methods [whisker MCB (circles: $T=0.27 \mathrm{~K}$ ), notched-wire MCB (squares: $T=0.036 \mathrm{~K}$ ), and thin-film MCB (triangles: $T=0.24 \mathrm{~K})]$. For comparison the expected behavior for tunneling- $I-V s$ between two BCS superconductors (lines) at the respective temperatures is shown. Inset: opening curve of a Zn-whisker MCB.

$(I-V)$ displayed in Fig. 2. It shows a well-pronounced superconducting gap of width $2 \Delta \simeq 320 \mu \mathrm{eV}$. For comparison we display $I-V s$ recorded on a so-called "notched-wire" break junction ${ }^{6}$ fabricated by a wire with diameter $125 \mu \mathrm{m}$ and one of a thin-film break junction ${ }^{11}$ together with the expected $I-V$ for a tunnel contact between two BCS superconductors. The gaps of the notched-wire and thin-film break junctions are smaller and the current onsets at $V=2 \Delta / e$ are shallower, although the data has been taken at lower temperatures.

Due to the nonideal hexagonal crystal structure (the ratio $c / a=1.82$ is larger than for $h c p$ ), the superconducting properties of $\mathrm{Zn}$ are anisotropic, with variations of the gap for different directions of the order of $20 \% .^{12}$ In the notchedwire and thin-film break junctions with a broad distribution of crystallographic directions, an average gap value with a smeared-out density of states is observed, while in the whisker only one direction is favored. The observed value is in the order of the expected value for the crystallographic $a$ axis, the direction with the largest gap. We can rule out the proximity effect with the In contacts $\left(2 \Delta_{\text {In }} \simeq 1.05 \mathrm{meV}\right)$ as reason for the enhanced gap, since the whisker is much longer than the superconducting coherence length (see below) and the breaking point is far away from the contacts.

The quality of the superconducting properties of the whiskers can further be elucidated by investigating $I-V s$ in an external magnetic field. The first-order transition to the normal state when applying a magnetic field perpendicular to the sample plane is hysteretic as expected for a type-I superconductor (see, e.g., Ref. 1) but hard to observe in bulk superconductors. The transition for raising the field is observed at $B_{c 1}=5 \mathrm{mT}$. From the supercooling of the normal phase down to $B_{c 2}=3.2 \mathrm{mT}$ when lowering the field, we determine a lower threshold of the coherence length of $\xi=\left(\phi_{0} / 2 \pi B_{c 2}\right)^{1 / 2} \simeq 320 \mathrm{~nm}$ (where $\phi_{0}$ is the superconducting flux quantum) in reasonable agreement with values from the literature. ${ }^{13}$ This large value of $\xi$ allows us to use $\mathrm{Zn}$ whiskers as electrodes for contacting nonsuperconducting systems such as semimetals, clusters, or molecules and to induce superconductivity via the proximity effect in order to analyze their transport properties along the lines of Ref. 14.

Figure 3(a) depicts a few examples for $I-V s$ of atomic contacts with total conductances in the order of $1 G_{0}$, reto AlP license or copyright, see http://apl.aip.org/apl/copyright.jsp 


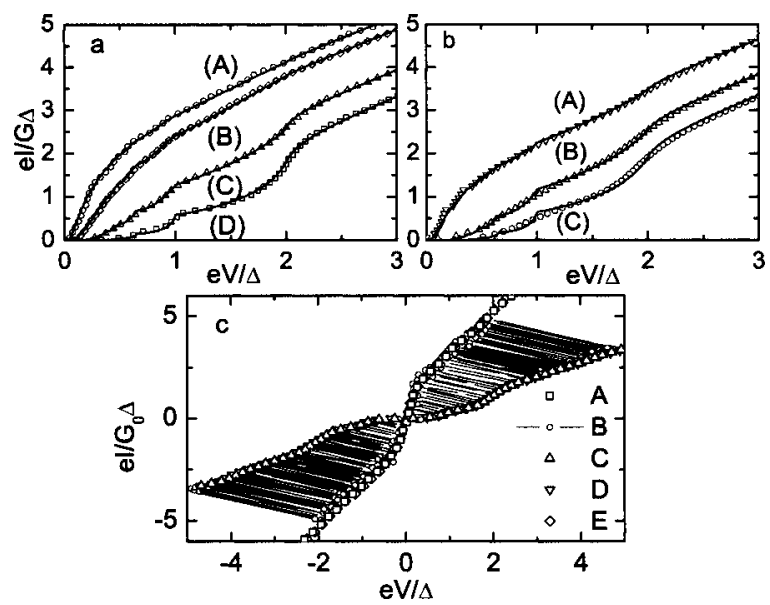

FIG. 3. (a) Current-voltage characteristics of several single-atom contacts of $\mathrm{Zn}$ arranged with a whisker $\mathrm{MCB}$ at $T=0.27 \mathrm{~K}$ (symbols) and best fits according to the theory of MAR (Refs. 15 and 16) (lines). The transmission coefficients are (A) $\tau_{1}=0.95$; (B) $\tau_{1}=0.86, \tau_{2}=0.01$; (C) $\tau_{1}=0.71, \tau_{2}=0.14$, $\tau_{3}=0.09$; and (D) $\tau_{1}=0.48, \tau_{2}=0.25, \tau_{3}=0.22$. (b) Respective data measured on a notched-wire break junction at $T<0.1 \mathrm{~K}$. Due to the rounding of the $I-V s$ a meaningful determination of the transport channels is not possible. Only a lower threshold $N_{\min }$ for the number of channels can be given. The total conductances and channel numbers are (A) $G=1.23 G_{0}, N_{\min }=3$; (B) $G=0.89 G_{0}, N_{\min }=2$; and (C) $G=0.75 G_{0}, N_{\min }=2$. The coefficients that have been used for calculating the solid lines are (A) $\tau_{1}=0.93, \tau_{2}=0.27$, $\tau_{3}=0.03$; (B) $\tau_{1}=0.67, \tau_{2}=0.22$; and (C) $\tau_{1}=0.48, \tau_{2}=0.26$. Different combinations of the $\left\{\tau_{i}\right\}$ give fits of equal quality. (c) Five I-Vs of a whisker MCB measured in the order (A)-(E). The transmission coefficients are (fits not shown): (D) $\tau_{1}=0.44, \tau_{2}=0.19$; (E) $\tau_{1}=0.96, \tau_{2}=0.47, \tau_{3}=0.09$. Only a reduced number of data points is shown for clarity.

corded on last plateaus before breaking the junction. Nonlinearities due to multiple Andreev reflection (MAR) (Refs. 15 and 16) are clearly discernable. Together with the data points we show the best fits to the theory of MAR from which the number and transmission coefficients of the transport channels of the contacts are deduced. ${ }^{17} \mathrm{Up}$ to four channels can be disentangled. In Fig. 3(b) we show for comparison a set of $I-V s$ recorded for contacts with similar resistances, but fabricated from a notched-wire break junction. Here it is impossible to determine the channel ensemble due to the systematic deviations between theory and experiment because of the rounded tunneling density of states. Thus, point contacts between single crystals with well-defined superconducting properties as, e.g., possible with whisker break junctions, are required when investigating the transport channels of anisotropic metals.

Finally, we address the precision and stability of the setup. Although the mechanical setup has a hysteresis of several micrometers for the position of the pushing rod, it is possible to switch repeatedly between two distinct configurations, as demonstrated in Fig. 3(c). It depicts five examples of $I-V s$ for contacts on the second-last and the last plateau before breaking the contact. The curves have been recorded in the order (A), (B), (C), (D), and (E). From curve (A) to (C) the contact has been opened. Then the contact was closed again. The agreement between the curves (A) and (E) [and between (C) and (D)] shows that those contacts have a very similar atomic configuration, thus demonstrating the reproducibility of particular atomic configurations of the constriction. The data taken at (B) fluctuate frequently (indicated by the thin line) between the two stable configurations formed by the pairs (A)-(E) and (C)-(D), respectively. The analysis of the channel ensemble reveals that configuration (C)-(D) is a single-atom contact with two transport modes while in configuration (A)-(E) three modes are active, corresponding presumably to a larger than a single-atom contact. However, since detailed calculations of the transport channels for larger contacts are still lacking, we cannot yet determine the exact atomic positions. While such a controlled arrangement of a particular atomic configuration only very rarely occurred with thin-film break junctions, ${ }^{18}$ we regularly observe such a behavior for the whisker break junctions.

In conclusion, we have developed a variant of the breakjunction technique that allows the fabrication of stable atomic-size and tunnel contacts with a purely mechanical drive. The electronic properties of the contacts are cleaner than those of contacts fabricated from notched-wire and thinfilm break junctions. It is thus possible to investigate important properties like the superconducting density of states for metals for which the standard fabrication methods of tunnel contacts do not give reliable results. The sharp and clean electrode tips of atomic size along with well-developed superconducting properties allow furthermore to contact other nanoobjects like molecules, or clusters for analyzing their transport channels. In the future, reliable breaking of the whisker in a suitable position will be improved by patterning the whisker with the help of a focused-ion beam.

The authors acknowledge enlightening discussions with C. Urbina, J. C. Cuevas, and R. Tidecks. P. B. and E. S. thank C. Urbina and R. Cron for their assistance and the CEA (France) for its hospitality during part of the measurements. This work was supported by the Deutsche Forschungsgemeinschaft through SFB 195, SFB 513, as well as the CFN, and by the Alfried Krupp von Bohlen and Halbach-Stiftung.

${ }^{1}$ M. Tinkham, Introduction to Superconductivity (McGraw-Hill, New York, 1996).

${ }^{2}$ R. Landauer, Philos. Mag. 21, 863 (1970).

${ }^{3}$ N. Agrait, A. Levy Yeyati, and J. M. van Ruitenbeek, Phys. Rep. 377, 81 (2003).

${ }^{4}$ E. Scheer, P. Joyez, M. H. Devoret, D. Esteve, and C. Urbina, Phys. Rev. Lett. 78, 3535 (1997).

${ }^{5}$ E. Scheer, N. Agraï, J. C. Cuevas, A. Levy Yeyati, B. Ludoph, A. MartínRodero, G. Rubio Bollinger, J. M. van Ruitenbeek, and C. Urbina, Nature (London) 394, 154 (1998).

${ }^{6}$ C. J. Muller, J. M. van Ruitenbeek, and L. J. de Jongh, Physica C 191, 485 (1992).

${ }^{7}$ U. Schulz, P. J. Wilbrandt, and R. Tidecks, J. Cryst. Growth 85, 472 (1987).

${ }^{8}$ U. Schulz, Ph.D. thesis, University of Göttingen, Germany, 1988

${ }^{9}$ A. I. Yanson, Ph.D. thesis, University of Leiden, The Netherlands, 2001.

${ }^{10}$ A. Mayer-Gindner, H. v. Löhneysen, and E. Scheer, cond-mat/0410054 (unpublished).

${ }^{11}$ J. M. van Ruitenbeek, A. Alvarez, I. Piñeyro, C. Grahmann, P. Joyez, M. H. Devoret, D. Esteve, and C. Urbina, Rev. Sci. Instrum. 67, 108 (1996).

${ }^{12}$ P. G. Tomlinson and J. C. Swihart, Phys. Rev. B 191867 (1979).

${ }^{13}$ C. Kittel, Introduction to Solid State Physics, 5th ed. (Wiley, Chichester, 1976).

${ }^{14}$ E. Scheer, W. Belzig, Y. Naveh, M. H. Devoret, D. Esteve, and C. Urbina, Phys. Rev. Lett. 86, 284 (2001).

${ }^{15}$ D. Averin and A. Bardas, Phys. Rev. Lett. 75, 1831 (1995).

${ }^{16}$ J. C. Cuevas, A. Martín-Rodero, and A. Levy Yeyati, Phys. Rev. B 54, 7366 (1996).

${ }^{17}$ M. Häfner, P. Konrad, F. Pauly, J. Heurich, J. C. Cuevas, and E. Scheer, Phys. Rev. B 70, 241404(R) (2004).

${ }^{18}$ E. Scheer, W. Belzig, D. Esteve, and C. Urbina, in Quantum Mesoscopic Phenomena and Mesoscopic Devices in Microelectronics, edited by I. O. Kulik and R. Ellialtioglu, NATO ASI Vol. 559 (Kluwer Academic Publishers, Dordrecht, 2000). 